\title{
A nuclear power plant accident in Fukushima: what should we do?
}

\author{
Seigo Kinuya
}

Published online: 12 November 2011

(C) The Japanese Society of Nuclear Medicine 2011

On March 11, 2011, several waves of huge tsunami after the biggest ever earthquake in the Japanese history hit the east areas of Japan and seriously damaged the nuclear power plant in Fukushima. Meltdowns in reactors followed by hydrogen explosion in the plant caused large-scale radioactive contamination to the environment. The concern of the health obstacle due to the radiation exposure spread through not only Japan but also the neighborhood nations. Although there is still a long way to go to provide the complete solution to the accidents, people are regaining their peace, thanks to the efforts at stabilization by the concerned parties struggling there.

Since the outbreak of accident, many scientific societies have been analyzing the data and the information disclosed by the government or the Tokyo Electric Power Company, Inc. (TEPCO) and announced statement of safety to the public. The Japanese Society of Nuclear Medicine (JSNM) launched a publicity campaign concerning thyroidal blockade, which was followed by several announcements regarding intake of contaminated foods, tap water, etc. (http://www.jsnm.org/japanese/11-06-02-0). This information was cited in a number of web sites including the governmental site of the Ministry of Health, Labour and Welfare. In addition, a lot of personal inquiries to the society office were quickly responded. Through these vigorous activities, JSNM were able to make contributions to provide people with accurate information.

However, it is still difficult to eliminate fear of radiation exposure from people. Nuclear medicine has been somewhat mistaken as being dangerous after the onset of this

S. Kinuya $(\bowtie)$

Department of Nuclear Medicine, Kanazawa University

Hospital, 13-1 Takara-machi, Kanazawa 920-8641, Japan

e-mail: kinuya@med.kanazawa-u.ac.jp accident. It has been reported that not a few people who were going to undergo targeted radionuclide therapies canceled them. The cancelation of the diagnostic tests such as FDG-PET studies has been reported, too. Facing to these incidents, JSNM had an additional announcement to stress the role and safety of nuclear medicine practices (http:// www.jsnm.org/japanese/11-04-21).

A paper of Higashi et al. [1] in this issue of Annals of Nuclear Medicine describes the situation of radioiodine therapy (RIT) for thyroid cancer in Japan with relation to the accident in Fukushima as well as the Japanese history since the atomic bombings in 1945 . The environment of RIT in Japan has been far from international standards: shortage of isolation rooms results in the difficulty to apply ablation and RIT to high-risk patients. Although a number of ablation and RIT increased from approximately 500 in 1987 to 3,000 in 2010 , there are still more than 3,000 patients untreated every year who are assumed to be therapeutic candidate for RIT. In contrast, the number of active isolation rooms decreased from 188 in 2002 to 138 in 2010. Because of these changes, patients recently should wait for longer than 6 months to undergo ablation or RIT after surgery. As shown by another paper of Higashi et al. [2] in Journal of Nuclear Medicine, this terrible condition would harm prognosis of patients with residual disease or distant metastases.

We took one big step toward the improvement of this situation. Ablation therapy for out-patients with $1,110 \mathrm{MBq}$ of ${ }^{131} \mathrm{I}$ was approved by the Ministry in November 2010. Up to July 2011, more than 200 patients underwent ablation at approximately 30 hospitals. Half of them were carried out at hospitals where isolation rooms are not installed. We are expecting that once out-patient ablation is widely performed to a number of patients without metastasis, isolation rooms will be efficiently 
utilized for patients with residual disease or distant metastases that require high dose RIT at 3.7-7.4 GBq or higher.

For the good growth of out-patient ablation, radiation safety should be secured. We should strictly follow the guidance of the Ministry and the guideline of JSNM and the related medical societies including Japan Radiological Society, Japan Thyroid Association, Japan Association of Endocrine Surgeons, Japanese Society of Thyroid Surgery, and Japanese Society of Nuclear Medicine Technology. According to the Basic Safety Standards (BSS) concept of the International Atomic Energy Agency (IAEA) and the guidelines regarding the release of patients after therapy with unsealed radionuclides (ICRP pub. 94), the Ministry requires to secure the dose limits of $1 \mathrm{mSv}$ per year to the public and $5 \mathrm{mSv}$ to caregivers of patients per episode for targeted radionuclide therapy. Out-patient ablation utilizes the largest amount of radioactivity in out-patient practices of nuclear medicine in Japan. Therefore, physicians are required to inform and educate patients in order to reduce radiation risk to their family and the public. Patients are asked not to go into the public during this period to minimize radiation exposure to others; that is, avoidance of use of the public transportation on the way back to home, no participation to public activities where people gather, etc. It is to be noted that hospitalization of patients in regular hospital rooms after their release from the dosing area or hotel stay of patients are generally not permitted because hospitals and hotels are essentially considered to be public spaces.

Furthermore, the development of out-patient ablation depends on understandings of the general public to acceptable amount of radiation exposure, because gamma exposure and environmental contamination to the public cannot be completely eliminated. Then, what is an acceptable dose? The dose $1 \mathrm{mSv}$ per year is enough? We have to keep in mind that the public sentiment against radiation exposure is completely changed after the outbreak of accident.

I would like to ask the readers of this editorial to imagine what will happen if the guideline is violated. A lot of people recently possess survey meters for radiation detection after the accident in Fukushima. They are checking radioactive contamination everywhere. What if they come close to patients? Some types of fire detectors equipped in public spaces are sensitive to radiation. What if gamma emission from patients may falsely activate them? We have to recognize that people would seriously oppose or claim against the release of patients due to these possible incidences in the current conditions.
As pointed out by Higashi et al. [1], Japanese people have feeling of radiophobia mainly due to the history of the atomic bombings. The contamination accident of a tuna fishing boat in 1954 and the Tokaimura JCO accident in 1999 are also attributable to this negative feeling. There is no doubt that the nuclear power plant accident in Fukushima evoked it again. Some people are too much afraid of slight amount of radiation exposure that is scientifically acceptable, partly because some media have been continuously releasing articles emphasizing that risks of low dose radiation are considerably high. If the people's feeling of radiophobia would grow worse and a strong opposition movement develops against the release of patients, the authority would not ignore them. Out-patient ablation would likely be suspended as a result. This scenario may not be limited to RIT, but may be extended to all matters of nuclear medicine. For instance, the adaptation of BSS for radioactive medical wastes might be abandoned if people object to it because of radiation risks.

The accident in Fukushima surely had a negative impact on the development of nuclear medicine. Now, we have to appeal to the public more than ever of the safety and usefulness of radionuclide application in practical medicine as well as experimental scientific fields. Open lectures and public programs concerning medical use of radiation may be helpful to ease the negative feelings of the general public. Collaboration with related societies utilizing radiopharmaceuticals will also be required in order to promote the understandings of people. It may be good to hold a cross-sectional scientific meeting to discuss this matter.

The authority requires that related societies should properly manage procedures regarding medical use of radiopharmaceuticals. We bear responsibility on securing radiation safety. I would really want all members of JSNM keep this in mind.

\section{References}

1. Higashi T, Kudo T, Kinuya S. I-131 Radioactive iodine therapy for differentiated thyroid cancer in Japan. Current issues with historical review and future perspective. Ann Nucl Med (in press).

2. Higashi T, Nishii R, Yamada S, Nakamoto Y, Ishizu K, Kawase S, et al. Delayed initial radioactive iodine therapy resulted in poor survival in patients with metastatic differentiated thyroid carcinoma: a retrospective statistical analysis of 198 cases. J Nucl Med. 2011;52:683-9. 